\title{
Effects of hue, brightness, contrast with background, and observation time on figural dominance in ambiguous patterns
}

\author{
FRANK S. MURRAY and MAUREEN V. RAGLAND \\ Randolph-Macon Woman's College, Lynchburg, Virginia 24503
}

\begin{abstract}
Ambiguous patterns composed of two alternate crosses of different hues and brightness on two different brightness backgrounds were viewed for $120 \mathrm{sec}$ by 10 female college students. Each subject observed 92 pattern presentations ( 23 patterns, each pattern presented in 2 orientations and on each of 2 backgrounds). Effects of hue and brightness contrast with background were cleariy demonstrated: blue was the most dominant, red the least, and green and yellow located in between. Brightness contrast of patterns with background accentuated figural dominance of the darker figures. The number of alternations increased over the observation time for hues of equal brightness; however, the relation of this measure to total duration of seeing a figure in studies of figural dominance is unclear. Theories of neural satiation, fatigue, and interaction were used in interpreting the results.
\end{abstract}

When people are presented with an ambiguous pattern (e.g., Rubin's or Köhler's crosses, "PeterPaul goblet"), they often report that the figure and ground alternate; that is, the part seen as figure becomes ground, and vice versa. The part of the pattern reported most often as figure, or that seems to dominate the viewing, is defined as showing dominance. Several studies have attempted to obtain quantitative data on the factors responsible for determining dominance (Brown, 1955; Cohen, 1959; Goldhamer, 1934; Graham, 1929; Harrower, 1936; Oyama, 1950; Price, 1967; Sadler \& Mefferd, 1970; Thetford, 1963). Some of the factors influencing figural dominance explored in these studies include hue, brightness, contrast with the surrounding background, and duration of observation time.

Graham (1929), using a variation of Köhler's cross, found a slight tendency for the red cross to dominate when both crosses in a red-blue pattern were of equal brightness. Later, Oyama (1950) compared a colored cross with a gray cross of the same brightness and found dominance to be a "decreasing function of the displacement of hue on both sides of red in the color circle" (p. 303). Therefore, red was the most dominant and blue the least dominant hue, with yellow and green falling between these two extremes.

The effects of brightness on figural dominance has led to controversial results. Graham (1929) found a tendency for the brighter red cross to dominate in a chromatic red-blue ambiguous pattern. However, this finding may be open to some criticism as the

The work on this paper was supported by a grant awarded to the first author from the Mednick Memorial Foundation. Requests for reprints should be sent to Frank S. Murray, Department of Psychology, Randolph-Macon Woman's College, Lynchburg, Virginia 24503. .effects of brightness were confounded with those of hue. Using achromatic ambiguous patterns, Goldhamer (1934) found increasing figural dominance with decreasing brightness, while Oyama (1950) obtained the opposite results. Many investigators have observed that "blackness" results in increased figural dominance in a black-white ambiguous pattern (Goldhamer, 1934; Graham, 1929). One possible explanation for black figural dominance is based on the acquisition of a set for perceiving black as figure. This acquired set would be formed from past experience with written or printed materials in which black letters are perceived as figure against a white background (Graham, 1929; Rock, 1975).

The brightness of the background which surrounds a visual pattern during presentation could also affect figural dominance. The results would differ depending on whether the pattern and the surrounding background were of unequal or of equal brightnesses (Harrower, 1936). If the pattern and the surrounding background were of unequal brightnesses, the appearance of the pattern as a whole would necessarily be influenced by the background due to simultaneous brightness contrast (Cornsweet, 1970; Haber \& Hershenson, 1973; Rock, 1975). For example, a bright background would tend to make a neutral gray pattern appear darker than if the same pattern were viewed against a dark background.

The duration of observation time as it affects the rate of apparent change (RAC) of a reversible pattern has been investigated by Brown (1955) and Cohen (1959). These investigators differed in their estimations of the time required to attain a maximum RAC, Brown (1955) placing the peak RAC after 2 min of observation time and Cohen (1959) placing the peak RAC after only 1 min of observation time. 
But both Brown (1955) and Cohen (1959) agreed that the RAC, plotted as a function of time, resulted in a negatively accelerated curve. Thus RAC was found to be an increasing function of time.

In a review of studies of reversible perspectives, Price (1969) contends that the lack of an adequate theory in this area may be due to the "haphazard methodology adopted by many workers in this area of research" (p. 102). According to Price, experimentation on reversible perspective is, in a large measure, characterized by the use of inefficient recording techniques and lack of adequate controls. A methodological improvement suggested by Price is the use of a continuous recording device during viewing so as to provide information about the RAC and the duration of each individual perceptual organization (initial time of viewing to see part of the pattern as figure). Using this technique, the total duration of $P_{1}$, the figural-dominating time of that part of the pattern which is first seen as figure, can be compared to the total duration of $P_{2}$, the figural-dominating time of that part of the pattern first seen as ground. Price also suggests the use of a tachistoscopic presentation of the visual pattern and a constant level of illumination during observation of the pattern.

The above suggestions were incorporated into the present study in an attempt to examine the effects of hue, brightness, contrast with surrounding background, and observation time on figural dominance in ambiguous patterns.

\section{METHOD}

\section{Subjects}

Ten female undergraduates volunteered to participate in the experiment. None of the subjects was color-blind, as tested by Dvorine Psuedo-Isochromatic plates.

\section{Procedure}

The stimulus materials consisted of 23 ambiguous patterns (Rubin's cross) constructed of Munsell matte paper. Each pattern was a circle, $7.5 \mathrm{~cm}$ in diameter, which was composed of eight $45^{\circ}$ sectors. The sectors alternated between two different hues, brightnesses, or combinations of both hue and brightness, to form two distinct crosses with each pattern. Six patterns were construcied from all possible pairings of the hues: red $(5.0 \mathrm{R} 5 / 6)$, green $(5.0 \mathrm{G} \mathrm{S/6)}$, blue $(5.0 \mathrm{~B} 5 / 6)$, and yellow $(5.0 \mathrm{Y} 5 / 6)$. These six patterns were equated along the brightness and saturation dimensions. These four hues were also paired with four brighter hues, 5.0 R 7/6, 5.0 G 7/6, 5.0 B 7/6, and 5.0 Y $7 / 6$, to produce 16 more patterns. Four of these patterns differed only in brightness, hue being held constant. The remaining 12 patterns differed in both hue and brightness. The last pattern was achromatic, consisting of white (N9/) and black $\mathrm{N} 2 /$ ) sectors. This pattern was included to provide an achromatic comparison to the four chromatic patterns which differed only in brightness.

Each of the 23 patterns was viewed against two surrounding backgrounds, either a gray (N5/) or a lighter gray (N7/). In order to control for a possible position effect, each pattern was viewed in two orientations, $22.5^{\circ}$ or $67.5^{\circ}$, in a clockwise rotation from the vertical axis. Using both backgrounds and orientations, there were four presentations of each pattern, for a total of 92 presentations.

The patterns were viewed through a Gerbrand's tachistoscope (Model G-1125). Illumination level on the pattern remained constant at $35 \mathrm{fc}$. (B 4 fluorescent lamps are used in this apparatus with a color temperature of $4,200 \mathrm{~K}$; the $x$ and $y$ coordinates on the ICI chromaticity diagram are $\mathrm{x}=.372, \mathrm{y}=.365$.) Each pattern was exposed for $120 \mathrm{sec}$. Prior to each presentation, a fixation fieid consisting of a small neutral gray dot centered within the $18.6 \times 18.6 \mathrm{~cm}$ viewing screen was exposed for $5 \mathrm{sec}$. The fixation field served to direct the subject's attention to the center of the screen before the stimulus pattern was presented. The subject's responses were recorded on a Gerbrand's continuous event recorder (Model P2-A).

After an example of an ambiguous pattern was given, the subject was instructed to press a switch and to indicate to the experimenter which color (cross) she saw during the viewing and to hold the switch on until the figure-ground organization changed and the second cross came into view. The subject was told to operate the switch appropriately as the pattern changed during the 120 -sec viewing time. For each pattern, the following measures were obtained: initial duration of viewing to see parts of the pattern as figure (organizational duration), the figure reported first, the number of (alternations), and the total duration of seeing figures. However, since organizational time was subtracted from the total duration measure, it was not used in any of the data analyses. Each subject received a randomly ordered sequence of the 92 patterns. Experimental sessions were approximately $30 \mathrm{~min}$, and were spaced over a 3-week period.

\section{RESULTS}

\section{Hue}

Of the six patterns in which different hues were presented, blue was seen first 85 out of 120 presentations (12 presentations to 10 subjects), green 72 times, yellow 52, and red 31 . The $D$ test developed by David (1963) was applied to these data for both background brightnesses. The $D$ test is a nonparametric analogue of the $F$ test for equality of the treatment means in analysis of variance and has been developed specifically for paired-comparisons designs. It provides a test of the hypothesis that there are no differences between the summed scores of $n$ judges for each of $t$ items contrasted with each other by the method of paired comparisons. $D$ is distributed approximately as chi square with $t-1 \mathrm{df}$. The results of the D tests for the four hues on the N5/ N7/ backgrounds were 22.1 and 20.2 , both significant for df $=3$ at $p<.005$. Post hoc comparisons revealed that for both backgrounds, blue was significantly different $(p<.05)$ from yellow and red, and that green was also significantly different from red. Similar results of figural dominance occurred for the total duration measure: blue dominated green, yellow, and red; green dominated yellow and red; and yellow dominated red. The results were similar for both background brightnesses. The two measures, proportion of times a figure was seen first and the proportion of total duration seeing a figure, were highly related, Spearman rank-order coefficient of correlation of .74. The mean number of alternations for the six patterns 
was 10.6: most alternations occurring to the R-G pattern $(\bar{X}=11.9)$, the least to the $R-Y$ pattern $(\bar{X}=9.8)$.

Analysis of variance for the six patterns at the two levels of background brightness over four $30-\mathrm{sec}$ intervals indicated significantly more alternations for patterns, $F(5,38)=15.78, p<.01$, and intervals $F(3,38)=3.38, p<.05$. No significant differences in alternations were obtained for background brightness. The mean number of alternations was highest for the R-G pattern (11.9) and least for the G-Y patterns (10.2). An increase in alternations was obtained for all patterns, except R-Y, over the 120 -sec interval.

\section{Brightness}

Of the four patterns in which brightness was varied and hue was constant, contrast with background brightness was the major factor in determining which figure was reported. With an N5/ background, the proportion of duration seeing the hue of $\mathrm{N} 7 /$ brightness exceeded the hue of N5/ brightness, and conversely with the hues of N5/ on the N7/ background (see Table 1). No significant differences were obtained in the total number of alternations for these four patterns.

With the achromatic pattern, black was reported seen first and of longer duration than white. Although responses were not significantly different to backgroung brightness, the proportion of the total duration seeing the black was accentuated to the brighter background: mean proportions of duration to black, .68 to N7/ background and .63 to N5/ background. No significant differences in alternations over the four 30-sec intervals were obtained. However, the mean number of alternations for 30 -sec interval was 12.3, which was higher than the chromatic patterns. If the RAC is a measure of perceptual stability of a pattern-that is, a low rate of alternation indicates less fluctuation of the figures and the pattern-then the achromatic pattern is less stable than the chromatic patterns used in this study.

\section{Hue and Brightness}

For those 12 patterns in which hue and brightness varied, the same dominance relationship was obtained. However, the effects of contrast of pattern with background were quite pronounced. The proportion of time the darker figure was seen increased significantly. Inspection of Table 1 shows, for example, that when a 5.0 B 5/6 was paired with a 5.0 R $7 / 6$ on a N7/ gray background, the proportion of the time blue was seen increased to .63 as to .49 when the same pattern was presented on a N5/ background. Table 1 also reveals that all the proportions of the hues of $5 / 6$ are at or below .50 on N5/ background and that all 16 of these hues exceed this value on the N7/ background. Assuming that the propor-
Table 1

Proportion of Total Duration Hue is Seen

\begin{tabular}{|c|c|c|c|c|c|c|c|c|}
\hline \multirow[b]{2}{*}{ 5/6 Hue } & \multicolumn{4}{|c|}{$\begin{array}{c}\text { N 5/ Background } \\
7 / 6 \text { Hues }\end{array}$} & \multicolumn{4}{|c|}{$\begin{array}{c}\text { N } 7 / \text { Background } \\
7 / 6 \text { Hues }\end{array}$} \\
\hline & B & $\mathbf{R}$ & $\mathbf{G}$ & $\mathbf{Y}$ & B & $\mathbf{R}$ & G & $\mathbf{Y}$ \\
\hline Blue & .48 & .49 & .48 & .49 & .61 & .63 & .65 & .62 \\
\hline Red & .45 & .41 & .46 & .47 & .60 & .58 & .67 & .60 \\
\hline Green & .41 & .46 & .48 & .42 & .61 & .62 & .66 & .58 \\
\hline Yellow & .50 & .43 & .44 & .45 & .58 & .61 & .62 & .58 \\
\hline
\end{tabular}

Note-These proportions are the total duration, excluding organization time, of the duration of dominance of the $5 / 6$ hues to the $7 / 6$ hues. $B=$ blue, $R=$ red, $G=$ green, and $Y=y$ ellow.

tion of duration one hue exceeds another hue is equally likely $(\mathrm{p}=.50)$, the binomial probability of such an occurrence of all 16 of the darker hues exceeding the lighter hues is $p<.00002$. No significant effects of hue were obtained for either background; the order of dominance was: blue, red, yellow, and green. Again, blue was the most dominant, but red, which was the least dominant when brightness of hues were equal, exceeded yellow and green in dominance when both hue and brightness were varied.

The mean number of alternations for the 12 patterns over the 120-sec observation period was significantly different, $F(11,80)=5.80, p<.01$; the smallest number of alternations occurring to the $5.0 \mathrm{G} 5 / 6$ vs. $5.0 \mathrm{R} 7 / 6$ pattern was 11.3 . As with total duration, the number of alternations to different background brightness was significantly different, $F(1,80)=17.74$, $p<.01$; the mean number of alternations were less for 10 of the 12 patterns on the brighter background N7/ than for the N5/ background. No significant differences in alternations occurred for the four 30 -sec intervals.

\section{DISCUSSION}

\section{Hue}

The effects of hue were clearly demonstrated in this study. Blue was the most dominant hue and red the least dominant, with yellow and green located between these two extremes. These results were supported by both of the measures of figural dominance, i.e., whether the cross was seen as figure first and whether the cross had a greater figural-dominating duration than the cross with which it was compared. These findings do not support the results of Oyama (1950). A possible explanation for the conflicting results is that Oyama (1950) compared a hue to a gray of the same brightness, and therefore made a chromatic-to-achromatic comparison rather than a straight hue-to-hue comparison.

\section{Brightness}

In terms of being seen as figure first, the darker 
cross in a chromatic pattern tended to dominate. Also, the black cross in the white-black pattern was always dominant. This result is consistent with the results of Goldhamer (1934) and Graham (1929). In the patterns composed of different hue/brightness combinations, the effects of hue seemed to be weaker than those of brightness since the darker cross usually dominated regardless of hue. The results of the figural-dominating duration for any one cross within a chromatic comparison which differed in brightness could not be explained by an absolute brightness effect. However, these results may be explained in terms of a brightness contrast effect (Lindauer \& Lindauer, 1970).

\section{Contrast with Background}

The figural-dominating duration of a cross within a chromatic comparison which differed in brightness seemed to be an increasing function of the amount of brightness contrast between that cross and the background-the Liebmann effect. This effect, as characterized by Vernon (1937), occurs when a figure which is relatively uncontoured and of the same brightness as the surrounding background tends to "blend" or "fuse" with the background. Such a blending or fusing process would seem to cause the remaining parts of the pattern to "stand out" more from the background, thereby increasing their figural dominance. Experimental evidence which would support the Liebmann effect had been obtained earlier by Harrower (1936), using a white-black ambiguous pattern. When the surrounding background was dark gray, the white cross dominated, and when the surrounding background was light gray, the black cross dominated.

\section{Duration of Observation Time}

The duration of observation time did have an effect on the RAC of patterns of the same brightness values, but not with patterns of different hues and brightness values. For those patterns which differed only in hues, the RAC appeared to increase with time of observation, which supperts the results of Brown (1955) and Cohen (1959) with reversible figures. No such relation occurred with the other ambiguous patterns used in this study. The meaning of RAC and its relation to other measures used in this experiment is unclear. If RAC is a measure of perceptual stability, one would expect that it should be inversely related to total duration; that is, when one part of the figure is seen for a substantial duration, then the pattern should produce fewer alternations. Spearman rank-order coefficients of correlation were -.03 and .08 for the six patterns on N5/ and N7/ backgrounds, which indicates almost no relationship between these measures. It appears from the results of this study that RAC and total duration are not related in measuring figural dominance of ambiguous patterns.

\section{Theoretical Interpretations}

The results of the present experiment could be subsumed under any of the theories of neural satiation, fatigue, or interaction (Attneave, 1971; Cornsweet, 1970; Ganz, 1966; Hochberg, 1950; Köhler, 1940). The classical theory of neural satiation by Köhler and Wallach (1944) proposed that the physiological basis of figure-ground reversal is found in the flow of electrical current alternating between two or more neural areas of the visual cortex-these neural areas isomorphically represent different figureground organizations. Electrical current in one neural area would produce an accumulation of resistance of satiation in that specific area. Satiation eventually reaches a critical threshold level which results in total inhibition of any further activity within the stimulated area. At this point, the electrical current would flow to a "fresh" (relatively unsatiated) neural area of the visual cortex which represents a second figure-ground organization, thereby producing an apparent figure-ground reversal to occur. In the case of a pattern with two possible figure-ground organizations, the current would return to the first neural area after the threshold level had once again been reached. If an adequate amount of time has not elapsed to allow the accumulated satiation in this area to fully decay, threshold would be reached more quickly. Therefore, RAC would increase as a function of time due to the decreased duration of each subsequent figureground organization. This prediction of the Köhler and Wallach satiation theory was confirmed in this experiment for patterns, of the same brightness values, but not for patterns of different hues and brightness values. Other psychological as well as physiological inconsistencies in the prediction of the Köhler and Wallach theory have been reported (Ganz, 1966; Hochberg, 1950; Kling \& Riggs, 1972).

One modification of the original satiation theory is offered by Attneave (1971). He proposes, as in the satiation theory, that the alternative aspects of a pattern are represented by activity in different neural structures, and that when one such structure becomes "fatigued" or satiated or adapted, it gives way to another that is fresher and more excitable. He further proposes that an analogous physical model of such an inhibitory process is the multivibrator flip-flop circuit in electronics.

Ganz (1966) has shown that satiation or fatigue theories have not accorded well with the experimental evidence from psychological and physiological studies. He has offered a quantitative model which incorporates lateral inhibition and contour inhibition to account for figure aftereffects. According to the 
theory, displacement occurs with figure aftereffects because the neural correlates of visual contour inhibit one another. Inhibition is proportional to contrast of contours to their background levels and is a decreasing function of the separation between the contours. It seems that the results of the present experiment on figure-ground dominance could be accounted for by such a theory or similar formulations.

\section{REFERENCES}

Atrneave, F. Multistability in perception. Scientific American, 1971. 225, 63.71.

Brown, K. T. Rate of apparent change in a dynamic ambiguous figure as a function of observation time. American Journal of Psychology, 1955, 68, 358-371.

COHEN, L. Rate of apparent change of a Necker cube as a function of prior stimulation. American Journal of Psychology, 1959, 72, $327-344$.

Cornsweet, T. N. Visual perception. New York: Academic Press, 1970.

DAvib. H. A. The method of paired comparisons. London: Griffin, 1963.

Ganz. L. Mechanisms of the figural aftereffects. Psychological Review, 1966, 73, 128-150.

Goldhamer, H. The influence of area, position, and brightness in the visual perception of a reversible configuration. American Journal of Psychology, 1934, 46, 189-206.

Graham, C. H. Area, color, and brightness difference in a reversible configuration. Joumal of General Psychology, 1929, 2 , 470-481.

HABER, R. N., \& HERShenson, M. The psychology of visual perception. New York: Holt, Rinehart, \& Winston, 1973.
HARRower, M. R. Some factors determining figure-ground articulation. British Journal of Psychology, 1936, 26, 407-424.

Hochaerg, J. E. Figure-ground reversal as a function of visual satiation. Journal of Experimental Psychology, 1950, 40, 682-686.

KIING, J. W., \& RIGgs, L. A. (Eds.). Woodworth and Schlosberg's experimental psychology (Vol. 1). New York: Holt, Rinehart, \& Winston, 1972.

KörLER, W. Dynamics in psychology. New York: Liveright, 1940.

Könleg, W., \& WAllach, H. Figural aftereffects: An investigation of visual processes. Proceedings of the American Philosophical Society, 1944, 88, 269-357.

Lindauer, M. S., \& LindauER, J. G. Brightness difference and the perception of figure-ground. Journal of Experimental Psychology, 1970, 84, 291-295.

OYAMA, T. Figure-ground dominance as a function of sector angle, brightness, hue, and orientation. Journal of Experimental Psychology, 1950, 60, 299-305.

Price, J. R. Two components of reversal rate for a rotating skeletal cube: "Conditioned satiation." Australian Journal of Psychology, 1967, 19, 261-270.

PRICE. J. R. Studies of reversible perspective: A methodological review. Behavior Research Methods \& Instrumentation. 1969, 1. 102-106.

Rock, 1. An introduction to perception. New York: Macmillan, 1975.

SADLER, T. G., \& MEFFERD, R. B., JR. Fluctuations of perceptual organization and orientation: Stochastic (random) or steady state (satiation). Perceptual and Motor Skills, 1970, 31, 739-749.

THETFORD, P. E. Influence of massing and spacing on Necker cube reversals. Perceptual and Motor Skills, 1963, 16, 215-222.

Vernon. M. D. Visual perception. London: Cambridge University Press. 1937.

(Received for publication January 2, 1976; revision accepted April 7, 1976.) 